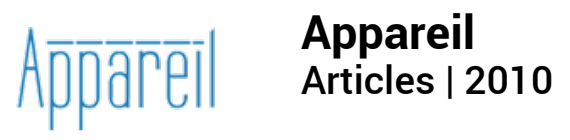

\title{
L'essence de la modernité selon Heidegger : la représentation
}

\author{
Marta Hernandez
}

\section{(2) OpenEdition}

Journals

Édition électronique

URL : http://journals.openedition.org/appareil/972

DOI : 10.4000/appareil.972

ISSN : 2101-0714

Éditeur

MSH Paris Nord

Référence électronique

Marta Hernandez, «L'essence de la modernité selon Heidegger : la représentation », Appareil [En ligne], Articles, mis en ligne le 08 mars 2010, consulté le 10 décembre 2020. URL : http:// journals.openedition.org/appareil/972; DOI : https://doi.org/10.4000/appareil.972

Ce document a été généré automatiquement le 10 décembre 2020.

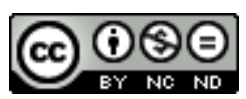

Appareil est mis à disposition selon les termes de la Licence Creative Commons Attribution - Pas d'Utilisation Commerciale - Pas de Modification 4.0 International. 


\title{
L'essence de la modernité selon Heidegger : la représentation
}

\author{
Marta Hernandez
}

1 Notre exposé prendra comme fil conducteur l'idée principale de Heidegger dans L'époque des conceptions du monde, à savoir que la représentation est la détermination de l'époque moderne. Nous mettrons en rapport cette idée avec la lecture heideggérienne de la Critique de la Raison Pure dans Kant et le problème de la métaphysique. Il nous a semblé que ce texte sur Kant, écrit en parallèle avec Être et Temps, éclaire d'avantage ce qu'Heidegger comprend par représentation en tant que fondement d'une époque. Pour cette raison il nous a aussi semblé que c'est à partir du Livre sur Kant qu'on pourrait mieux cerner la critique d'Heidegger sur la modernité. Le second propos de ce détour par Kant, ne pourra qu'évoquer très superficiellement le sujet plus vaste où s'inscrit notre recherche concernant la pensée d'Heidegger. Ainsi, le thème qui, dans notre réflexion a commandé le passage par la question de la représentation chez Heidegger, ainsi que par la Critique kantienne, est celui de la khôra du Timée de Platon à qui Heidegger consacre quelques lignes dans l'Introduction à la métaphysique. Dans la mesure où Heidegger voit dans la khôra platonicienne la préfiguration de l'espace cartésien, il nous semble légitime de supposer que la khôra du Timée est aussi la préparation pour l'avènement de la représentation dans la modernité. Notre hypothèse, qu'on se contentera aujourd'hui juste d'énoncer, c'est qu'il est possible de tracer une analogie entre le rôle accordé par Heidegger aux formes a priori de la sensibilité chez Kant et celui attribué à la khôra du Timée, lieu où pour Heidegger s'opère pour la première fois la séparation (en grec khôrismós) de l'être et de l'étant.

Dans L'époque des conceptions $d u$ monde, Heidegger fait de la représentation la détermination de l'époque moderne: "Que l'étant devienne étant dans et par la représentation, voilà ce qui fait de l'époque qui en arrive là une époque nouvelle par rapport à la précédente. $»^{1}$ Si l'on définit couramment "époque » comme un temps marqué par un événement, la représentation est pour Heidegger «la marque déterminante de la modernité. » 
3 Cela veut dire, d'ores et déjà, que la représentation n'est pas un facteur parmi d'autres qui viendrait compléter l'analyse historiographique de la modernité. La science historique (la historia) présuppose la représentation en ceci qu'elle considère le fait historique comme un objet. "Dans les sciences historiques aussi bien que dans les sciences naturelles, le procédé vise à représenter ce qui est constant (beständing) et de faire ainsi de l'histoire un objet (Gegenstand). Or, l'histoire ne peut devenir objet de la représentation que si elle est passée. Ce qui dans le passé est constant, c'est-à-dire ce au compte de quoi l'explication historique peut porter l'unique et le multiple de l'histoire, c'est ce qui a toujours déjà été (das Immer-schon-einmal-Dagewesene) l'ensemble du comparable. $»^{2}$ Ainsi, bien plus qu'un trait ou caractère qu'on puisse isoler et/ou mettre en rapport avec d'autres afin de tracer le profil d'une époque, la représentation est pour Heidegger la base (le fondement: grund) des Temps Modernes. Et ceci dans un double sens : la représentation est en même temps la manière générale où l'époque moderne se comprend elle-même et le fondement qui détermine la loi et la méthode pour les différents savoirs particuliers qui ont comme visée une région du monde. S'il est ainsi, la représentation doit être mise au clair comme étant la manière dont, dans la modernité, l'être de l'étant se détermine à chaque fois (dans un champ de savoir délimité, qu'il soit scientifique, historique, politique, etc.) et, plus largement, comme étant la condition sine quoi none de «la conception moderne du monde.» À suivre Heidegger, cette double exigence, expression d'un rapport non dialectique entre le général et le particulier, devrait expliquer à elle seule les phénomènes essentiels des Temps Modernes. Ceux-ci sont pour Heidegger la science, la technique mécanisée, "l'entrée de l'art dans l'horizon de l'esthétique ", « l'interprétation "culturelle" de tous les apports de l'histoire humaine », et le « dépouillement des dieux ».

4 Cette totalisation quant à l'ampleur de la représentation, lui confère d'avantage sa portée ontologique. Le questionnement heideggérien vers la représentation fait surgir celle-ci comme le champ préalable de la pensée moderne. C'est à partir de l'idée de champ préalable qu'Heidegger peut faire comparaitre dans un même plan (dans une même consistance quant à son fondement) les oppositions acquises de la métaphysique. Or, ce qui est acquis premièrement, dans tous les cas, ne sont pas ces oppositions en tant que telles. Celles-ci seraient bien fondées par la pensée scientifique qui s'assure par ce procédé «critique » de son opérabilité. Le sens du critique ainsi que le rapport du «critique» au champ préalable, renvoie à ce que dit Heidegger dans Concepts fondamentaux de la philosophie antique : "Critique : krinein - "scinder", "différencier", en différenciant quelque chose de quelque chose au sein d'un couple, rendre visible ce qui est différencié et ce qui le différencie. Différencier: un triangle d'un carré, un mammifère d'un oiseau, l'épopée du drame, le substantif du verbe, un étant d'un autre étant. $\|^{3}$ En réalité, toutes les sciences opèrent et déterminent ce genre de distinctions. De ce fait, les sciences positives sont "critiques », mais elles le sont d'une toute autre manière que la philosophie. La différence essentielle réside dans l'élaboration de leurs visées : la spécificité du champ de la philosophie fait de cette dernière, selon Heidegger, la science critique. Car ce qui est véritablement critique est ce que les sciences, à la vue de leur rigueur, s'interdisent de penser, à savoir : le champ préalable aux oppositions réglées entre les étants.

Ces oppositions sont le résultat d'une scission où l'étant est élaboré comme objet. Objectivation et représentation vont ensemble. « Re-présenter - dit Heidegger - signifie ici : faire venir devant soi, en tant qu'ob-stant [...] ce qui est là-devant [...], le rapporter 
à soi, qui le représente, et le ré-fléchir dans ce rapport à soi en tant que région d'où échoit toute mesure $»^{4}$. C'est ainsi que la représentation est explicitée par Heidegger premièrement comme un certain rapport à soi. La région, où la représentation comme mesure invariable de l'être à l'étant viendrait à échec, est celle du dasein. Le dasein est en lui-même rapport à l'êtrecomme le support d'une expérience de soi autrement que représentative. C'est dans la mesure où le dasein s'expérimente lui-même dans la mouvance de sa coappartenance à l'être qu'il est constamment en rapport mobile à celui-ci, l'être en tant qu'être étant mouvement (kínesis) et de ce fait non susceptible d'être objectivé, voire représenté. C'est en ce sens que l'être en tant qu'être est dépassement de l'étantité au même titre que le dasein est dépassement de la subjectivité. Dans ce mouvement réciproque du da-sein au sein, fondant du rapport de co-appartenance de l'être et de l'étant, la position de la pensée consiste pour Heidegger à se tenir dans l'ouverture à l'être, à savoir dans la question. Or, la question se meut toujours dans un champ préalable, qui lui sert d'orientation. Dans ce sens, la représentation serait le champ préalable aux oppositions métaphysiques, aussi bien qu'à la propre question heideggérienne sur l'être, car c'est dans le champ ouvert par la métaphysique que cette question se rapporte au fondement de celle-ci, comme à son présupposé ontologique. La différence réside dans la manière de se prendre à la représentation. C'est cette manière qui élabore la visée propre à chacune. Pour la métaphysique, ce champ préalable est un acquis non questionné, qui laisse dans l'obscurité le fondement de l'étant en tant qu'étant, c'est-à-dire la manière où l'étant est élaboré comme une objectité. De ce fait, l'être même tombe sous le coup de la représentation aussi bien que le néant: pour la métaphysique, l'être est compris soit comme la totalité des étants, soit comme ce que l'étant n'est pas, c'est-à-dire dans son opposition à lui ; et le néant, ce qui n'est pas ni l'être ni l'étant de manière absolue, à savoir ce qui n'est pas représentable. C'est pourquoi, dans la mesure où le fondement de l'objectité comme élaboration de l'étant dans la métaphysique n'est pas mis en lumière, la question sur l'être ne peut se poser que comme la question sur l'étant, à savoir « Pourquoi donc y a-t-il l'étant et non pas plutôt rien ? ${ }^{5}$

6 En revanche, la manière proprement philosophique ou proprement critique consiste à questionner le champ préalable d'apparition des phénomènes afin de dévoiler ses présupposés ontologiques. Car, qu'on le sache ou pas, selon Heidegger il est toujours question d'une ontologie, c'est-à-dire d'une pré-compréhension de l'être. Celle-ci est déterminée par avance d'une certaine manière, on l'a dit, en tant que la compréhension s'oriente à l'intérieur d'un champ préalable qui lui est transcendant; la transcendantalité du champ préalable constitue en même temps la source de toute individuation. ${ }^{6}$ Que la métaphysique ait masqué la question sur ce préalable sous la forme de la question sur l'étant, (c'est-à-dire qu'elle n'ait pas posé directement la question sur l'être), ne reste rien au fait que ce qui est en jeu dans une telle "conception du monde» (à savoir du monde comme objectité) est le rapport de l'humain à l'être. " Reste encore la possibilité de placer la problématique de la réalité en deçà de toute orientation selon un "point de vue", en défendant la thèse : tout sujet est ce qu'il est seulement pour un objet et vice versa. Mais dans cette position de départ toute formelle, les membres de la corrélation restent aussi indéterminés ontologiquement que celle-ci. Au fond, la corrélation tout entière devient bel et bien nécessaire comme étant "d'une certaine manière", elle est donc pensée en référence à une certaine idée d'être. $»^{7}$ 
7 Dans la perspective de dégager les enjeux qui sont impliqués dans une telle critique à la modernité comme époque de la représentation, le texte d'Heidegger sur Kant, « Kant et le problème de la métaphysique ", permet d'avantage de cerner la problématique du rapport entre la représentation comme mode d'apparition de l'être de l'étant dans la modernité et la question ontologique. La position paradoxale qu'occupe Kant dans la pensée heideggérienne présente l'avantage d'être explicitée par Heidegger comme telle. Cette position n'est pas, dans un certain sens, différente de celle attribuée par Heidegger aux différents noms propres de l'histoire de la philosophie (Platon, Aristote, Hegel, Nietzsche, Husserl) qui semblent être considérés par lui tantôt comme des représentants de la métaphysique (c'est-à-dire de la pensée représentative) tantôt comme précurseurs de la problématique posée par Être et Temps. Selon nous, cette dualité, dont la pensée kantienne devient la référence vis-à-vis de la logique immanente à la pensée d'Heidegger, n'est autre que la dualité même de la pensée, projection de la duplicité originaire de l'être et de l'étant.

Dans « Kant et le problème de la métaphysique », Heidegger part du questionnement de la représentation comme fondement de la métaphysique pour arriver à la découverte du temps comme transcendance. Bien que pour Heidegger il s'agisse du même chemin de la pensée, nous allons mettre en relief l'idée que deux parcours implicites dans la démarche heideggérienne peuvent être suivis séparément. Nous allons juste suivre très superficiellement le premier. Celui-ci concerne l'instauration chez Kant de la « représentation en général » en tant que fondement ontologique de la connaissance de l'être de l'étant par et dans la subjectivité. Dans ce sens, le texte sur Kant pose les bases de l'analyse critique de la modernité qui sera complétée et élargie par Heidegger dans des textes postérieurs tels que «L'époque des conceptions du monde » et "Dépassement de la métaphysique ». Le deuxième parcours suit la découverte d'Heidegger dans la Critique de la Raison Pure de la temporalité constitutive de la raison finie. Dans le croisement de ces deux parcours, se joue pour Heidegger l'ambivalence de la position kantienne: Kant, ayant ouvert le champ pour la compréhension de la question de l'être comme temporalité s'interdirait de penser la question de la médiation en termes de structures existentielles du dasein du fait d'avoir pris - dit Heidegger - la « position de Descartes ».

9 Le point sur lequel nous voulons insister concernant le texte sur Kant est celui du parallélisme dans le discours d'Heidegger entre les structures ontologiquesexistentielles du dasein dans Être et Temps et les représentations pures de l'espace et le temps dans Kant et le problème de la métaphysique. C'est ainsi que la question de la représentation peut être posée dans son rapport au fondement de l'ontologie. La représentation ne peut se constituer comme structure d'apparition de l'être de l'étant que parce qu'elle occupe dans la modernité le rôle médiateur accordé aux existentielles du dasein dont la seule élaboration était déjà d'une certaine manière l'élaboration de la question sur l'être. À suivre Heidegger, la représentation, phénoménologiquement élaborée, occuperait la même place ("position» de l'être en tant qu'être, au sens d'Heidegger) dans la Critique de la Raison Pure que celle occupée par les structures ontologique-existentielles du Dasein dans Être et Temps. Il s'agit bien, dans les deux cas, du lieu donnant accès à l'être de l'étant, c'est-à-dire celui des structures phénoménales d'être, auxquelles la pensée critique doit constamment se rapporter.

Le propos du «Livre sur Kant» est en partie annoncé dans Être et Temps: «Dans l'horizon de la problématique kantienne, ce qui se conçoit phénoménologiquement 
comme phénomène peut être illustré, sous réserve d'autres distinctions, en disant : ce qui déjà se montre dans les apparitions (Erscheinungen), ce qui chaque fois, fût-ce de manière non thématique, précède et accompagne le phénomène dans son entente courante, peut être amené à se montrer de manière thématique et ce qui se-montreainsi-par-soi-même ("les formes de l'intuition"), ce sont des phénomènes de la phénoménologie. $»^{8}$ Les formes de l'intuition, l'espace et le temps, sont pour Kant des représentations pures a priori, c'est-à-dire des représentations de la forme en général dont les objets se présentent à la conscience. En ce qui concerne la connaissance chez Kant, la présence de l'objet à la conscience dans l'intuition pure n'est pas une pure donnée sensible car les conditions de réception du phénomène dans la sensibilité ont la forme la plus générale (la plus pure) de la représentation. Ainsi, l'acte de réception du phénomène est une élaboration spatio-temporelle sous la forme de la représentation, ce qui implique un espace et un temps élaborés eux-mêmes de manière aprioristique comme des représentations. À cette représentation de l'étant (du phénomène au sens de Kant) dans l'intuition pure, l'entendement ne viendrait donner sa forme définitive (la forme du concept comme représentation de l'entendement) qu'après ce premier coup où l'étant est élaboré comme objectité dans l'intuition: «On sous-estime trop facilement ce que dit Kant dans la première phrase du corps de l'exposé de la Critique de la Raison Pure - dit Heidegger -: "De quelque manière et par quelque moyen qu'une connaissance puisse se rapporter à des objets, le mode par lequel elle se rapporte immédiatement aux objets et que toute pensée prend comme intermédiaire pour les atteindre [...] est l'intuition." »"

Connaître veut dire pour Kant premièrement intuitionner..$^{10}$ On se rappellera à ce propos que dans Être et Temps, Heidegger, qui veut se démarquer essentiellement de la vision théorétique de la phénoménologie husserlienne, dit que la factivité (« caractère d'être du Dasein inhérent à l'existence » et qu'on découvre dans la disposibilité ${ }^{11}$ ) « ne saurait se rencontrer dans une intuition ». Pour Kant (et l'on pourrait ajouter aussi pour Husserl) c'est le contraire qui se passe : la pensée est au service de l'intuition, car c'est dans celle-ci que sont élaborés les phénomènes comme représentations de la sensibilité. Les formes pures a priori de l'intuition sont les représentations pures de la forme en général où les phénomènes sont perçus, c'est-à-dire ce sont des représentations sans objet. Celles-ci, ancrées dans la subjectivité, permettent l'accès à l'être de l'étant pour la conscience. Sans cette médiation, le phénomène n'apparaîtrait tout simplement pas en tant que tel (c'est-à-dire en tant que ceci ou cela) pour un sujet. Dans la mesure où la question de la médiation est pour Heidegger celle de la connaissance du rapport en général de l'être et de l'étant, Heidegger ramène le problème de l' "Esthétique transcendantale " chez Kant à celui de la connaissance ontologique, c'est-à-dire de la connaissance de la détermination en général de l'être de l'étant. Étant donné que le mode d'apparition des phénomènes est enraciné chez Kant dans l'intuition pure, Heidegger rapporte à celle-là la vérité ontologique de la «Critique » à laquelle la vérité ontique (c'est-à-dire la connaissance par concepts) doit, dit Heidegger, nécessairement se conformer. En recadrant ainsi la question du fondement de la Raison Pure, Heidegger veut mettre à l'épreuve le point de vue selon lequel la Critique de la Raison Pure, comme théorie de la connaissance, serait l'aboutissement de l'esprit critique de la modernité. Ainsi, dit Heidegger, "l'interprétation de la connaissance comme acte de juger (penser) fait violence au sens décisif du problème kantien. $»^{12}$ Ce qui serait «critique » alors, au sens d'Heidegger, c'est le domaine où s'opère le krinein ou la séparation entre ce qui est phénomène et ce 
qui ne l'est pas, c'est-à-dire entre ce qui est et ce qui n'est pas représentable. Ce domaine c'est bien celui du fond ou fondement sur lequel sont posées les bases de l'édifice ou architectonique de la raison, car c'est dans celui-là où réside la possibilité même de la critique comme théorie de la connaissance métaphysique, cette dernière comprise comme le développement de ses possibilités intrinsèques. C'est alors la représentation en général qui trace le cadre de la critique : du bas, les représentations pures de l'espace et le temps, condition de possibilité de la connaissance d'un objet en général, vers le haut, les idées de la raison, en tant que celles-ci constituent les limites de la connaissance par concepts en franchissant les limites de la représentation.

C'est ainsi que la séparation critique est déjà inscrite comme représentation dans "le "plan préalablement projeté" d'une nature en général ». Celle-ci « détermine, d'emblée, la constitution de l'être de l'étant, auquel toute recherche et toute question doivent pouvoir se rapporter. ${ }^{13} C^{\prime}$ 'est de cette manière que le fondement de la théorie de la connaissance est rejeté vers le fondement de la connaissance ontologique, c'est-à-dire de la connaissance des structures phénoménales d'être qui rend possible la manifestation de l'étant comme tel. «Si la pensée est ainsi essentiellement relative à l'intuition, l'une et l'autre doivent posséder une certaine affinité permettant leur union mutuelle. Cette affinité, cette origine dans un même genre (genus) s'expriment en ce que, pour toutes deux, "représentation en général" (repraesentatio) est le terme générique $»^{14}$

13 Le mot latin repraesentatio désigne dans la Critique de la Raison Pure la "représentation en général ", terme générique qui rassemble les différentes représentations (vorstellung) ou synthèses représentatives qui ont lieu dans la conscience. La repraesentatio kantienne renvoie à ce que Heidegger dit dans L'époque des conceptions $d u$ monde : « Par rapport à l'entente grecque, la représentation moderne signifie tout autre chose. Cette signification s'exprime le plus clairement dans le mot de repraesentatio ". Ce que Heidegger veut dire ici c'est que le mot qui exprime le mieux la pensée représentative moderne en la différenciant de la pensée grecque est repraesentatio et non pas Vorstellung.Le verbe vorstellen veut dire pareillement représenter et présenter. Il comprend stand (position), c'est-à-dire la position occupée par ce qui est posé là devant. L'être posé là devant se rapporte au représenter (vorstellen) comme à sa « mise en position " (stellen), celle-ci étant le fond de permanence où l'objet se tient comme "l'unité de la position stable (ständigkeit) ». ${ }^{15} \mathrm{La}$ double signification du verbe «vorstellen » reflèterait pour Heidegger l'erreur de la modernité, à savoir le fait de prendre la représentation de la chose par la pure présence d'une donnée sensible à la conscience. Ceci est d'autant plus confondant que pour les grecs l'être est présence. Autrement dit : pour les grecs il y aurait une sorte d'indifférenciation ou indistinction entre la chose (ce qui apparaît comme étant là devant) et son champ d'apparition ou champ préalable. En revanche, la perception de soi supposée par l'ego cogito cartésien (inaugurateur de la modernité), n'est pas fondée selon Heidegger sur la simple présence à soi mais sur la représentation de soi comme chose pensante. Si bien la «représentation en général " (la repraesentatio) comme mode d'apparition de l'être de l'étant (c'est-à-dire comme pré-compréhension de l'être) était déjà le champ préalable de la formulation cartésienne de l'ego cogito, à suivre Heidegger il aurait fallut attendre Kant pour que la représentation se dévoile dans sa transcendantalité, c'est-à-dire pour que la représentation se présente à nous avec les traits propres à l'être. C'est de cette manière que, dans son analyse phénoménologique de la représentation, Heidegger élève celle-ci au rang d'être de la modernité : elle serait à la fois le plus transcendant 
vis-à-vis des objets (le sujet devenu transcendantal dans sa coïncidence avec la pure forme de la représentation en général); et le plus individualisant en tant que représentation de l'objet par le sujet empirique, ce qui constitue du même coup le sujet comme représentation de soi. Tout se passe comme si pour Heidegger la vorstellung comprise comme (re)présentation empirique de la conscience se rapportait à la « représentation en général » (repraesentatio) en tant que " genre » d'être de l'étant. Ce que Heidegger, à la suite de Kant, comprend par repraesentatio introduit une tournure dans la citation d'Heidegger qui nous a servi d'ouverture à cet exposé. Selon cette tournure Heidegger voudrait dire: "Que l'étant devient étant dans et par la repraesentatio ou représentation en général, celle-ci étant le "genre" d'être de l'étant dans sa coïncidence avec la forme générale de la subjectivité, voilà ce qui fait de l'époque moderne une époque nouvelle par rapport à la précédente. » Ainsi, la question de la représentation en général déplacerait la question de la subjectivité empirique vers celle de la coïncidence de la subjectivité et de la représentation dans le champ préalable d'apparition des phénomènes. Il est ainsi que la subjectivité, lieu de la représentation en général, serait, dans l'époque moderne, à la base de la subjectivation aussi bien que de l'objectivation. «Sans doute les Temps Modernes ont-ils, par suite de l'émancipation de l'homme, amené le règne d'un subjectivisme et d'un individualisme. Mais il est tout aussi certain qu'aucune époque avant les Temps Modernes n'a produit un objectivisme comparable, et qu'en aucune époque précédente le non-individuel n'a eu tant d'importance, sous la forme du collectif. L'essentiel à retenir ici, c'est le jeu nécessaire et réciproque entre subjectivisme et objectivisme. Or, précisément, ce conditionnement réciproque renvoie à des processus plus profonds. »

14 Jaques Derrida, qui dans la conférence publiée sur le titre Envoi, discute la traduction heideggérienne de repraesentatio par vorstellung, ne fait pas allusion au fait que cette « équivalence » serait due plus à Heidegger lecteur de Kant qu'à Heidegger lui-même. En réalité, la traduction du mot latin repraesentatio par l'allemand vorstellung remonterait à Wolf, chez qui la vorstellung fait déjà référence à la représentation comme forme de la connaissance intellectuelle. Le tournant opéré par Wolf par rapport à Leibniz consiste en ce que le signifié, qui était la fonction principale de la monade leibnizienne du point de vue de la forme, voire de sa représentation, est transposée et restituée dans la faculté intellectuelle. C'est ainsi que le sens de la vorstellung, comme représentation de la conscience, apparaîtrait pour la première fois. Heidegger, chez qui le gros de la critique à la modernité consiste à dévoiler la manière dont les modernes ont pris la représentation de la chose par la pure présence de la chose à la conscience, s'appuie sur Kant pour déplacer la question de la représentation de la conscience individuelle vers celle de la représentation comme mode général d'apparition de l'être de l'étant. Par ailleurs, dans la Critique de la Raison Pure, Kant utilise pareillement les mots de repraesentatio et de vorstellung pour se référer à la "représentation en général ", mais il réserve le terme vorstellung pour nommer les représentations de la perception et de l'entendement. Dans la mesure où Kant n'utilise le mot repraesentatio que pour désigner la «représentation en général», Heidegger aurait préféré le mot latin pour mettre l'accent sur le fait qu'en tant que fondement la repraesentatio transcende l'acte empirique subjectif de la perception et de l'entendement.

Cette insistance concernant la traduction historique et pas seulement heideggérienne de la repraesentatio latine par la vorstellung allemande, corrobore d'avantage l'argumentation derridienne. Ce que Derrida veut dire dans la conférence citée, c'est que dans la mesure où Heidegger ne questionne pas l'équivalence entre la repraesentatio 
et la vorstellung,il se situe d'emblée dans la tradition métaphysique. Cette appréciation à propos du non questionnement d'Heidegger vis-à-vis de la vorstellung n'est pas sans importance, étant donné que la question de l'interprétation heideggérienne de la philosophie grecque ainsi que l'élaboration conceptuelle de sa propre pensée doit beaucoup à une traduction qui voudrait être non représentative, c'est-à-dire non métaphysique. C'est parce que selon Heidegger la métaphysique implique un certain rapport à la langue qu'il peut, par exemple, attribuer la distinction entre le substantif et le verbe au Sophiste de Platon, et préciser en l'occurrence qu'il ne s'agit pas d'une distinction grammaticale mais métaphysique.

Selon Derrida, la préférence d'Heidegger pour le mot re-praesentatio, afin de souligner la différence entre la pensée moderne et la pensée grecque, repose sur le fait que le re- de la repraesentatio implique une valeur qui est étrange à la vorstellung. Re-praesentatio veut dire rendre présent. Le « rendre » présent, par rapport à la pure présence (praesentatio) incorpore la possibilité de la répétition, à savoir de pouvoir faire venir à la présence quelque chose et cela de manière répétitive, c'est-à-dire dans la temporalité du retour, ce qui ne serait possible que sur l'hypothèse d'une présence supposée. "Dans la réprésentation, le présent, la présentation de ce qui se présente revient, fait retour comme double, effigie, image, copie, idée en tant que tableau de la chose désormais disponible, en l'absence de la chose, disponible, disposée et prédisposée pour, par et dans le sujet. Pour, par et dans, le système de ces prépositions marque le lieu de la représentation ou de la Vorstellung. Le re-marque la répétition dans, pour et par le sujet, a parti subjecti, d'une présence qu'autrement se présenterait au sujet sans dépendre de lui ou sans avoir en lui son lieu propre. ${ }^{16}$

Nulle représentation ne serait pour Heidegger que subjective et en même temps la subjectivité est déjà l'élaboration de la représentation, comme représentation de soi. Jusqu'à l'instant on a essayé d'élucider la question de la représentation chez Heidegger suivant sa lecture de la Critique de la Raison Pure. Selon cette perspective, la subjectivité transcendantale serait le lieu de la représentation en général (repraesentatio) et la subjectivité empirique serait le lieu de la vorstellung comprise comme représentation pour et dans une conscience. On a voulu montrer que l'élaboration phénoménologique de la représentation et de la subjectivité à partir de la lecture de Kant est le préalable pour ainsi dire dont on ne peut pas se passer pour comprendre l'allure de la critique d'Heidegger à la modernité en tant qu'époque éminemment représentative.

Cependant, les limites de notre interprétation sont posées par Heidegger dans ce même texte, L'époque des conceptions du monde. "L'homme grec est en tant qu'il est l'entendeur de l'étant; voilà pourquoi le monde, pour les Grecs, ne saurait devenir image conçue (Bild). En revanche, que pour Platon l'étantité de l'étant se détermine comme eidos (adspect, "vue") voilà la condition lointaine, historiale, souveraine dans le retrait d'une secrète médiation, pour que le Monde (Welt) ait pu devenir image (Bild). ${ }^{17}$ Cette allusion à Platon comme pré-figurateur de la pensée représentative interroge de fond en comble la manière dont, dans la pensée d'Heidegger, la représentation se constitue comme la détermination d'une époque. La représentation est plus largement et d'avantage chez Heidegger la détermination de la métaphysique en tant qu'envoi et accomplissement de l'être. D'ailleurs, Heidegger n'a jamais enfermé sa critique à la représentation dans le cadre de sa critique à la modernité. Bien plutôt, sa critique à la représentation est la critique de la pensée envers elle-même, c'est-à-dire envers son champ préalable en tant que celui-ci contient ses possibilités de développement aussi 
bien que la possibilité de son propre dépassement. C'est dans cette perspective qu'Heidegger interroge la métaphysique dans l'Introduction à la métaphysique, texte où la représentation est au commencement des quatre scissions donnant lieu au commencement de la pensée occidentale, à savoir les scissions être et apparence, être et devenir, être et penser et être et devoir. Ces quatre scissions nous renvoient à l'idée que la représentation est déjà comprise en tant que possibilité dans la duplicité originaire de la pensée.

19 "Quand nous disons "Être", cela veut toujours dire: "Être de l'étant". Quand nous disons "l'étant", cela veut dire : l'étant eu égard à l'Être. Nous parlons toujours du sein de la duplicité. Celle-ci est toujours donnée déjà d'avance, aussi bien pour Parménide que pour Platon, aussi bien pour Kant que pour Nietzsche. La duplicité a déjà déployé le domaine à l'intérieur duquel la relation de l'étant à l'être devient représentable. Cette relation se laisse interpréter et expliquer de différentes façons. ${ }^{18}$

Il est ainsi que la représentation se retrouve différemment dans trois moments de l'histoire de la pensée occidentale dont le trait d'union est celui de l'histoire comme Geschichte (destin ou envoi) :

1. un premier moment dans l'origine de la pensée où la possibilité de la représentation est déjà comprise dans la duplicité de l'être et de l'étant ;

2. un deuxième moment dans le commencement de la métaphysique où la représentation est rendue possible par le khôrismós de Platon, c'est-à-dire par la séparation de l'être et de l'étant;

3. un troisième moment dans l'époque moderne, où la représentation en général s'instaure comme fondement.

Ces trois moments marqueraient trois commencements : le commencement originaire de la pensée, où la représentation ne serait comprise que comme possibilité et se tiendrait à l'état de latence; le commencement de la pensée occidentale, voire de la métaphysique, celle-ci étant la fin de la pensée classique grecque et la préparation de l'avènement de la modernité; le commencement de la modernité par l'ego cogito cartésien.

Conçue dans son rapport à la pensée platonicienne, la représentation chez Heidegger déborde les limites de notre interprétation basée sur le texte sur Kant, dans la mesure où elle déborderait le cadre tracé par la pensée critique d'Heidegger. Ce cadre, où il faut situer Heidegger dans sa polémique avec le néo-kantisme, est celui de la transcendance de la représentation en général et de son ancrage dans la subjectivité. Par rapport à ce " champ préalable ", on trouvera dans la philosophie de Platon le « contrepoint », pour ainsi dire, d'une pensée (celle d'Heidegger) qui par son essence critique, serait renvoyée incessamment au champ préalable qui la rend possible. Parce que, d'une part, Heidegger souligne à plusieurs reprises le fait que les grecs n'ont pas l'idée d'un espace représentatif dans la mesure où pour eux la chose n'est pas séparable du lieu propre à la chose. D'autre part, les lignes consacrées par Heidegger à la khôra du Timée dans l' Introduction à la métaphysique veulent «indiquer - dit Heidegger - qu'à partir de la philosophie platonicienne, dans l'interprétation de l'être comme idéa, se prépare la transformation par laquelle l'essence à peine soupçonnée du lieu (topos) et de la khôra, se substitue l'espace défini par l'extension ${ }^{19}$. En même temps, l'hypokeimenon grec, que le latin traduit par subjectum, n'aurait rien à voir selon Heidegger avec le sujet moderne. On soulignera à notre propos que nulle part Heidegger n'affirme que l' hypokeimenon grec puisse être compris comme l'antécédent ou pré-figurateur de la 
subjectivité moderne. Cela voudrait dire, qu'il n'est pas question ici ni d'une analogie entre l'hypokeimenon grec et le sujet moderne, ni d'un trait "commun» dont l'historialité du dasein pourrait rendre compte. Pour nous la question réside en ceci que, selon Heidegger, la représentation moderne est égale à la subjectivité et la pensée de Platon préfigure cette représentation moderne; pourtant dans la pensée de Platon, selon Heidegger, on ne trouvera pas les traces de la subjectivité. «Le décisif, ce n'est pas que l'homme se soit émancipé des anciennes attaches pour arriver à lui-même, mais que l'essence même de l'homme change, dans la mesure où l'homme devient sujet. Ce mot de subjectum, nous devons à la vérité le comprendre comme la traduction du grec hypokeimenon. Ce mot désigne ce qui est étendu-devant [...], qui, en tant que fond (Grund), rassemble tout sur soi. Cette signification métaphysique de la notion de sujet n'a primitivement aucun rapport spécial à l'homme et encore moins au "je" ».20

En conclusion, l'élaboration phénoménologique de la représentation met Heidegger dans une position paradoxale vis-à-vis de sa propre pensée. D'une part, la représentation ne saurait être la détermination de l'époque moderne que parce qu'elle coïncide dans sa forme générale avec la subjectivité transcendantale. D'autre part, dans la mesure où le commencement de la métaphysique coïncide avec le commencement de la représentation dans la pensée de Platon, la représentation transcenderait les limites de la subjectivité et serait la détermination de la métaphysique, même dans les larges périodes historiques où l'on ne trouverait, à suivre Heidegger, aucune trace de la subjectivité.

Pourvu que l'élaboration phénoménologique de la représentation se situe dans le cadre de la question ontologique, à savoir que la question vers la détermination historiale de l'être, ce même mouvement questionnant vers la genèse d'une telle pensée ne peut que s'arrêter là où la pensée de l'autrui (Platon dans ce cas-ci) devient dis-conforme à une telle interprétation. Nulle part Heidegger ne s'exprime plus clairement par rapport à sa méthode d'interprétation que dans la $4^{\mathrm{e}}$ préface du Livre sur Kant: «C'est ainsi que la problématique d'Être et Temps intervînt en tant que préconçu en l'interprétation tentée de Kant. Le texte de Kant devînt l'asile de la recherche en Kant d'un partisan de la question de l'être posée par moi. Un tel asile mène à ce que la Critique de la Raison Pure fût interprétée du point de vue de la problématique d'Être et Temps et en vérité d'une problématique qui lui est étrangère bien qu'elle la conditionne, fût attribuée à la question de Kant. »

On pourrait supposer, et il est légitime, que la pensée d'Heidegger sur Platon est aussi une projection de la propre problématique heideggérienne et en finir là. Pourtant deux choses nous interdiraient de considérer la lecture heideggérienne de Platon en parallèle avec sa lecture de Kant. La première c'est que, on ne peut pas lire l'interprétation heideggérienne de Platon en ce qui concerne le commencement de la métaphysique à la lumière d'Être et Temps. En effet, dans Être et Temps il n'y a pas un seul mot contre Platon et quand Heidegger s'y réfère, il le fait la plupart du temps par rapport au couple Platon-Aristote, en tant que représentants de la pensée classique grecque. Dans la même perspective, dans Kant et le problème de la métaphysique, Heidegger attribue à Kant, en tant que précurseur d'Être et Temps, le privilège d'être, parmi tous les modernes, le seul à entrer directement en discussion avec Platon et Aristote. En réalité, la pensée d'Heidegger se fixe face à Platon à partir des années trente, au moment où la critique à l'époque moderne s'intensifie. Cette fixation est liée à la question du commencement de la métaphysique et de son achèvement dans 
l'époque moderne, par opposition au commencement originaire de la pensée. Étant donné que l'époque moderne est non seulement l'aboutissement de la métaphysique mais aussi sa fin historiale, c'est-à-dire la clôture de la métaphysique comme système représentatif, la question du commencement attribué à Platon doit être lue à la lumière du déclin de la modernité et de l'annonce d'une nouvelle période où l'être, après vingttrois siècles d'oubli dans et pour la métaphysique (à savoir dans et pour la représentation), apparaîtrait pour la première fois: «En même temps que la métaphysique entre dans la période de son achèvement commence la préparation, inconnue, essentiellement inaccessible à la métaphysique, d'une première apparition du Pli de l'être et de l'étant. Dans cette apparition se cachent encore les premières lueurs de la vérité de l'être, laquelle retire en elle la primauté que l'être possède en ce qui concerne sa puissance. $»^{21}$

\section{NOTES}

1. M. Heidegger, "L'époque des "conceptions du monde" ", dans Chemins qui ne mènent nulle part, Paris, Gallimard, 1962, p. 118.

2. M. Heidegger, "L'époque des "conceptions du monde" ", dans Chemins qui ne mènent nulle part, Paris, Gallimard, 1962, p. 108.

3. M. Heidegger, Concepts fondamentaux de la philosophie antique, Paris, Gallimard, 2003, p. 19.

4. M. Heidegger, "L'époque des "conceptions du monde" ", dans Chemins qui ne mènent nulle part, Paris, Gallimard, 1962, p. 119.

5. M. Heidegger, «La question fondamentale de la métaphysique», dans Introduction à la métaphysique, Paris, Gallimard, 1967.

6. M. Heidegger, Etre et Temps, Paris, Gallimard, 1986, §7.

7. M. Heidegger, Être et Temps, $\$ 43$.

8. M. Heidegger, Être et temps, §7.

9. M. Heidegger, Kant et le problème de la métaphysique, Paris, Gallimard, 1981, p. 83.

10. M. Heidegger, Kant et le problème de la métaphysique.

11. M. Heidegger, Être et temps, § 29.

12. M. Heidegger, Kant et le problème de la métaphysique, p. 84 .

13. M. Heidegger, Kant et le problème de la métaphysique, p. 71.

14. M. Heidegger, Kant et le problème de la métaphysique, p. 84.

15. M. Heidegger, "Dépassement de la métaphysique", dans Essais et conférences, Paris, Gallimard, 1958, p. 84.

16. J. Derrida, « Envoi » dans Psychè. Inventions de l'autre, Paris, Galilée, 1987, p. 120-121.

17. M. Heidegger, "L'époque des "conceptions du monde" ", dans Chemins qui ne mènent nulle part, p. 119.

18. M. Heidegger, Qu'appelle-t-on penser ?, Paris, PUF, 1959, p. 261.

19. M. Heidegger, Introduction à la métaphysique, p. 50.

20. M. Heidegger, "L'époque des "conceptions du monde" ", dans Chemins qui ne mènent nulle part, p. 115.

21. M. Heidegger, « Dépassement de la métaphysique » dans Essais et conférences, p. 89-90. 